\title{
Micromechanical modelling of porous viscoelastic composite materials
}

\author{
Mohamed El Kouri, ${ }^{1, a}$, Abderrahmane Bakkali ${ }^{1,2}$ and Lahcen Azrar ${ }^{1,3,4}$ \\ ${ }^{1}$ Modeling and Control, Department of Mathematics, Faculty of Sciences and Techniques of Tangier, AEU, Tangier, Morocco \\ ${ }^{2}$ Department of Physics, Faculty of Sciences of Tetouan, Abdelmalek Essaadi University, Tetouan, Morocco \\ ${ }^{3}$ Research Centre STIS, team M2CS, Department of Applied Mathematics and Informatics, ENSET, MV University, Rabat, Morocco. \\ ${ }^{4}$ Department of Mechanical Engineering, Faculty of Engineering, KAU, Jeddah, Saudi Arabia
}

\begin{abstract}
Modelling and predicting the effective behavior of non-ageing viscoelastic composites have attracted the attention of many researchers. Actually, predicting the effective behavior and the macroscopic overall response while taking into account the constituents properties and shape of inclusions as well as their volume fractions is a challenging topic. In this work, porous viscoelastic composites are considered. The porous effect is introduced as a solid voided inclusions embedded in a viscoelastic matrix. The effective behavior is modelled by delayed integropartial differential equations. The resolution of the resulting equations is done through a methodological approach based on the Volterra tensorial products and the dynamic Green's tensor. Thus, the localization equations relating the local and the global fields are derived. After that, the Mori-Tanaka mean field micromechanical model assumptions are applied to derive the Mori-Tanaka's localization tensor. Once this step is completed, the effective properties are obtained through mean field techniques. The effective properties are given through tensorial convolution products. A numerical algorithm is elaborated for the computation of direct and inverse tensorial convolution products. For the validation of the developed modelling a comparison with Laplace-Carson approach is done.
\end{abstract}

\section{Introduction}

Porous viscoelastic materials have been widely used due their wide range of applications: acoustic absorption, vibration isolation, low thermal conduction, electricity and magnetism shielding, as well as fire isolation. Many works have been done to model and investigate the ageing and non ageing viscoelastic materials. Two kinds of micromechanical approaches have been developed: The classic one that provides the effective behavior in the Laplace domain then a numerical inversion is performed to the time one and there is the approach that provides the effective behavior directly in the time domain.

Eshelby micromechanical models for elasticity have been extended, based on the Laplace transform, to the case of viscoelasticity by many researchers. Laws and McLaughlin [1] estimated the effective behavior of viscoelastic composites based on the Self consistent model. In the numerical results, attentions were given to the case of spherical and fibrous composites. Wang and Weng [2] extended the Mori-Tanaka model to examine the linearly viscoelastic behavior of composites with randomly oriented inclusions and of transversely isotropic ones with aligned spheroidal inclusions. Afterwards, Brinson and Lin [3], and Fisher and Brinson [4], among others, analyzed the viscoelastic behavior of viscoelastic composites using different micromechanical models. The Mori-Tanaka model was extended to the case of viscoelastic composites with interphase regions.
The effect of shape of inclusions, volume fractions, and thickness of the interphase region were shown on the effective behavior. This approach based on the Laplace transform was also extended to analyze the behavior of coupled field composite materials. Jiang and Batra [5] derived closed form expressions in time and frequency domain of viscopiezoelastic composites consisting of parallel PZT cylinders of elliptic cross section embedded in a viscoelastic matrix. Azrar et al. [6] and Bakkali et al. [7] extended the Laplace transform approach to predict the time and frequency dependent behavior of viscopiezoelectric and viscomagnetoelectroelastic composite materials.

The purpose behind developing the second approach that derives the effective behavior directly in the time domain is to overcome the limitation of the first one: The case when the composite is subjected to thermomechanical loading, or one of the phases constituted the composite present an ageing behavior.

Lahellec and Suquet [8] predict the non ageing effective behavior of viscoelastic composites based on an internal variable approach. The developed modelling was reduced to a system of non linear equations at each step of time. Ricaud and Masson [9] developed a modelling based on the internal variable approach to predict ageing and non ageing viscoelastic behavior of viscoelastic composites.

The effective behavior was obtained by the resolution of a system of linear equations at each step of time. This

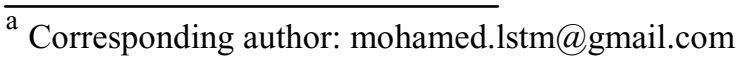


work was extended by [10] to predict the ageing behavior of polycrystals. A displacement formulation was developed by Sanahuja [11] to investigate the ageing behavior of viscoelastic composite constituted of spherical inclusions.

Recently, El Kouri et al. [12] developed a new formulation based on The Dynamic Green's function techniques and on the integral equations allowing the prediction, directly in time domain, of non ageing viscoelastic composites, in the general case of anisotropic viscoelastic composites with ellipsoidal inclusions. More recently, Barthélémy et al. [13] extended the Eshelby inclusion problem for elasticity to ageing viscoelastic composites. The case of isotropic behavior with spherical inclusions and time independent Poisson ratio is considered in the numerical section.

In this work, the effective behavior and averaged fields per phase of non ageing viscoelastic composites with voided inclusions are investigated. The presented modelling gives straightforward expression of the effective behavior, in the time domain, in the general case of anisotropic viscoelastic composites with ellipsoidal voided and non voided inclusions.

This work is organized as follows: First, the governing equations are presented. Second, based on the dynamic Green's functions and on the integral equations the concentration tensors are derived. In the next section, The Mori-Tanaka mean field approach is presented and the effective properties are derived. The numerical algorithm allowing the calculation of the direct and inverse tensorial convolution products is also presented. Finally, in the numerical section, a comparison is done between the prediction obtained based on the Laplace approach and the one presented in this paper. Effective properties, global and local response are presented for different cases. The effect of volume fraction, shape of voided inclusions is shown on the behavior of the considered viscoelastic composites.

\section{Governing equations}

In this work, voided viscoelastic composites constituted of voided inclusions embedded in a viscoelastic matrix are considered. A representative volume element (REV) $\Omega$ is considered. At the boundary $\partial \Omega$, the macroscopic strain evolution $E(t)$ is linked with the displacement $u(x, t)$ by the following relationship $u(x, t)=E(t) \cdot x$. The non ageing behavior at each $x \in \Omega$ is modelled by a Stieltjes integral relating the stress tensor evolution $\sigma(x, t)$ to the strain tensor history $\varepsilon\left(x, t^{\prime}\right)$ as follows

$$
\sigma_{i j}(x, t)=\int_{0}^{t} C_{i j k l}\left(x, t-t^{\prime}\right) \frac{\partial \varepsilon_{k l}\left(x, t^{\prime}\right)}{\partial t^{\prime}} d t^{\prime} \quad \forall x \in \Omega
$$

where $C_{i j k l}\left(x, t-t^{\prime}\right)$ is the relaxation tensor.

A compact form of Equation (1) is given by the following convolution product in the Stieltjes space

$$
\sigma_{i j}(x, t)=\left[C_{i j k l}(x)^{*} d \varepsilon_{k l}(x)\right](t) \quad \forall x \in \Omega \forall t>0
$$

The compatibility equations, when the small displacement are considered, are given by the following expression

$$
\varepsilon_{i j}(x, t)=\frac{1}{2}\left[u_{i, j}(x, t)+u_{j, i}(x, t)\right] \quad \forall x \in \Omega
$$

where $u_{i}(x, t)$ is the displacement components. In the absence of the body force, one can write the equilibrium equations as follows

$$
\operatorname{div}[\sigma(x, t)]=0 \quad \forall x \in \Omega
$$

Considering the symmetry of the tensors $\sigma, \mathcal{E}, C$ and based on the previous equations, one can derive the following system of partial differential equation

$$
\left[C_{i j k l}(x)^{*} d u_{k, l}(x)\right]_{, j}(t)=0 \quad \forall x \in \Omega
$$

\section{Integral equations and localization}

Let consider a homogeneous fictitious media which has the relaxation moduli $C^{0}(t)$. The local relaxation moduli is given by the following expression

$$
C_{i j k l}(x, t)=C_{i j k l}^{0}(t)+\delta C_{i j k l}(x, t)
$$

in which $x$ is the position vector in the considered media and $\delta C$ is the deviation part.

Using the above perturbation equation, based on the dynamic Green's tensor and after some mathematical development the following localization equation is obtained [12]

$$
\varepsilon_{m n}^{0}(t)=\left[\left(I_{m n k l} H(t)-\frac{1}{\Omega_{I}}\left[T_{m i j} * d C_{i j k l}^{0}\right](t)\right) * d \varepsilon_{k l}^{I}\right](t)
$$

with $T_{m n i j}(t)$ is the dynamic viscoelastic interaction tensor given by:

$$
T_{m n i j}(t) \int_{\Omega_{I}} \int_{\Omega_{I}} \Gamma_{m n i j}\left(x-x^{\prime}, t\right) d \Omega^{\prime} d \Omega
$$

In this paper, the Mori-Tanaka model, presented in the next section, is considered. The derived concentration tensor in this section is the main step of the micromechanical modelling. The difficulty behind the calculation of the concentration tensor is that a direct and an inverse tensorial convolution product has to be computed. For this reason, a numerical algorithm is developed.

\section{Effective properties and numerical algorithm}

The Mori-Tanaka mean field approach is one of the most used micromechanical models. It is known by its accurate predictions, easy to implement and gives explicit expression of the effective properties. The expression of 
the time dependent Mori-Tanaka localization tensor is obtained by replacing $\varepsilon^{0}(t)$ and $C^{0}(t)$ of the reference media in equation (7) by the evolution strain $\varepsilon^{M}(t)$ and relaxation moduli $C^{M}(t)$ of the matrix. Therefore, one can write the following relationship relating the local evolution strain in the voided inclusion with the evolution strain in the matrix through the so called the dynamic localization tensor

$$
\varepsilon_{k l}^{I}(t)=\left[A_{k l m n}^{I(M T)} * d E_{m n}\right](t)
$$

where $A_{k l m n}^{I(M T)}$ is the Mori-Tanaka dynamic localization tensor and its expression is given by

$$
A_{k l m n}^{I(M T)}(t)=\left(H(t) I_{m n k l}-\frac{f_{M}}{\Omega_{I}}\left[T_{m n i j} * d C_{i j k l}^{M}\right](t)\right)^{-1}
$$

where $f_{M}$ is the volume fraction of the matrix and $\Omega_{I}$ is the volume of the voided inclusion.

Now that the localization tensor is derived, average techniques are used to obtain the effective properties and overall behavior.

The overall behavior of the composite is modelled by

$$
\Sigma_{i j}(t)=\left[C_{i j k l}^{e f f} * d E_{k l}\right]
$$

Based on average techniques [12], the following expression of the effective properties is obtained

$$
C_{i j m n}^{e f f}(t)=C_{i j m n}^{M}(t)-f^{I}\left[C_{i j k l}^{M} * d A_{k l m n}^{I(M T)}\right](t)
$$

The average evolution strain in the matrix is obtained through the following expression

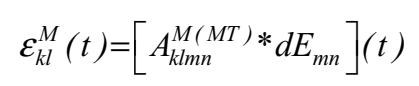

$A_{k l m n}^{M(M T)}$ is obtained from the equation that verifies the localization tensors

$$
f^{M} A_{k l m n}^{M(M T)}+f^{I} A_{k l m n}^{I(M T)}=H(t) I_{k l m n}
$$

Based on these equations, one can predict the overall and local behavior of the considered viscoelastic composite for different shape of inclusions. Various laws modelling the behavior of the viscoelastic matrix could be considered.

The main difficulty of this work is the calculation of the direct and inverse tensorial product. The algorithm is based on the discretization of time through on a trapezoidal algorithm [12]. Based on this trapezoidal algorithm, one can transform the convolution product in the Stieltjes space to a matrix-vector product as follows

$$
\left[\Sigma_{i j}\right]_{(n+1)}=\left[C_{i j k l}^{e f f}\right]_{(n+1) \times(n+1)}\left[E_{k l}\right]_{(n+1)}
$$

where $\quad\left[\Sigma_{i j}\right]_{(n+1)}$ and $\left[E_{k l}\right]_{(n+1)}$ are vectors of $(n+1)$ elements and $\left[C_{i j k l}^{e f f}\right]_{(n+1) \times(n+1)}$ is a $(n+1) \times(n+1)$ matrix.

More details about this algorithm are given in [12]. The presented numerical algorithm allows one to compute the Mori-Tanaka estimate of two phase viscoelastic composites in the general case of ellipsoidal inclusion and anisotropic behavior.

\section{Numerical results}

In this section, the numerical results of viscoelastic composites consisting of voided inclusions embedded in a non ageing viscoelastic matrix are presented. The viscoelastic matrix is considered to be isotropic. The bulk $K$ and the shear $G$ moduli of the viscoelastic matrix are described by the Prony series.

$$
G(t)=G_{\infty}+\sum_{j=1}^{N} G_{j} \exp \left(-\frac{t}{\xi_{j}}\right)
$$

where $G_{\infty}$ is the rubbery asymptotic modulus. $G_{j}$ and $\xi_{j}$ are the relaxation moduli and the relaxation time. The used Prony series coefficients are given in [12].

For the sake of comparison, the results predicted based on the Laplace transform approach and on the presented modelling are compared. More details about the Laplace transform approach could be found in [12]. Based on this approach and the developed modelling for a non ageing linear viscoelastic composite consisting of spherical voided inclusions embedded in a viscoelastic matrix, Figure 1 gives the predicted results for the volume fraction of void $f^{I}=0.3$. One can see that the two approaches predict the same results.

Based on the developed modelling for a viscoelastic composite consisting of voided inclusions embedded in a viscoelastic matrix and for the volume fraction of void fixed at $f^{I}=0.2$, Figure 2 gives the effective moduli as function of the different shapes of voided inclusions. The effect of the later is shown on the behavior of the considered composites.

For the particular case of spherical voided inclusions, Figure 3 illustrates the effect of the void volume fraction on the effective moduli.

Now to show the global and local response of the considered viscoelastic composites, the following macroscopic uniaxial strain is applied

$$
E_{11}(t)= \begin{cases}10^{-4} t ; & t<5 \mathrm{~s} \\ 5 \times 10^{-4} ; & t \geq 5 \mathrm{~s}\end{cases}
$$

In Figure 4, a viscoelastic composites constituted of spherical voided inclusions embedded in a viscoelastic matrix is considered. The global and local response of the composites when it is subjected to the uniaxial strain is presented for different void volume fractions. 


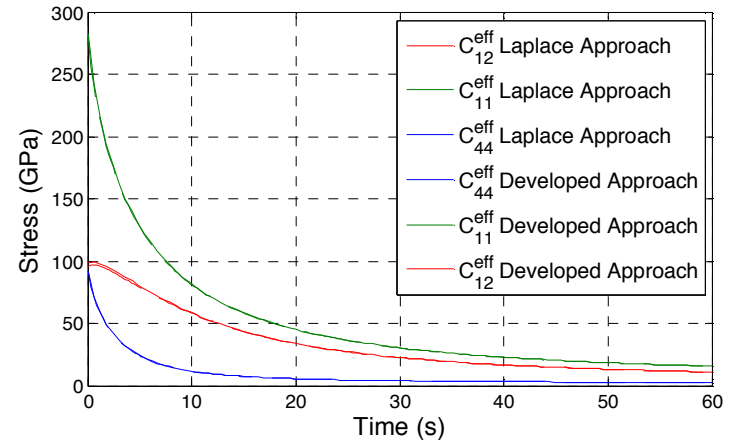

Figure 1. Effective moduli $C_{12}, C_{11}$ and $C_{44}$ as predicted by Laplace transform and non ageing linear viscoelastic model.

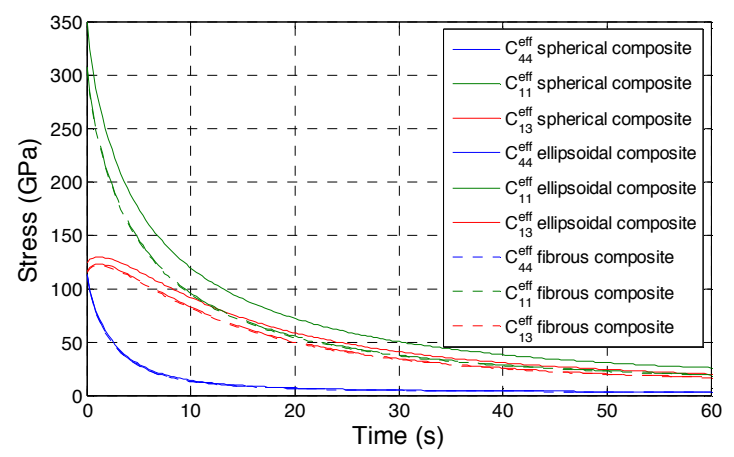

Figure 2. Effective moduli $C_{44}, C_{11}$ and $C_{13}$ as function of the shape of voided inclusions.
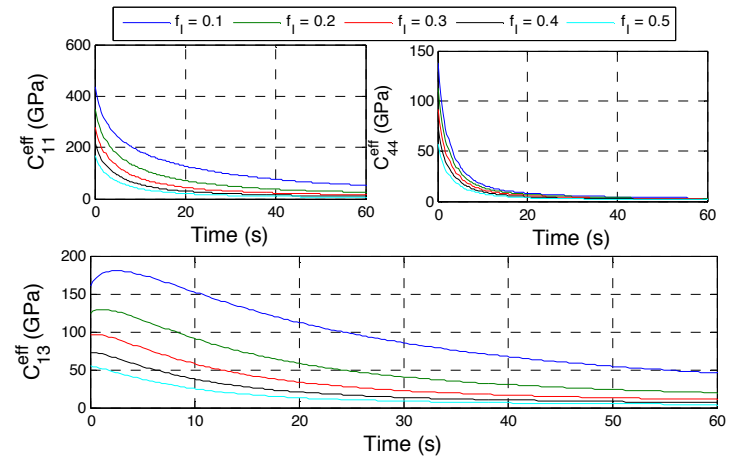

Figure 3. Effective moduli $C_{11}, C_{44}$ and $C_{13}$ as function of the void fraction for spherical voided inclusions.

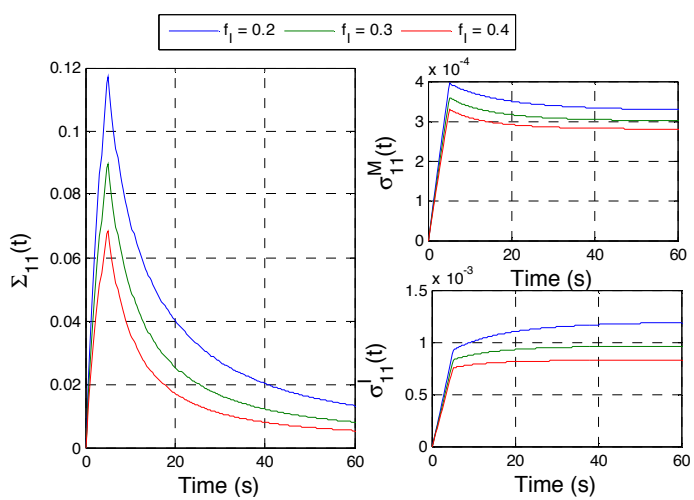

Figure 4. The global and local response of a viscoelastic composite, consisting of voided inclusions embedded in a viscoelastic matrix, subjected to uniaxial strain.

\section{Conclusions}

In this paper a micromechanical modelling based on the dynamic Green's functions and the Mori-Tanaka mean field approach is presented to analyze the effective behavior of viscoelastic composites constituted of voided inclusions.

The presented modelling permits a straightforward derivation of the effective behavior in the time domain. The difficulty of this work lies in the fact that a direct and inverse convolution tensorial product has to be calculated.

A numerical scheme is presented for this purpose. In the numerical section, a viscoelastic composite consisting of voided inclusions embedded in a viscoelastic matrix is considered. A comparison between the developed modelling and Laplace transform approach is presented. Different effective moduli are presented and the effect of the volume fraction and the shape of the voided inclusions is shown on the them. The global and local response is also investigated when the considered composite is subjected to a uniaxial strain.

\section{References}

1. N. Laws, R. McLaughlin, Proceedings of the Royal Society of London A: Mathematical, Physical and Engineering Sciences 359, 251 (1978)

2. Y.M. Wang, G.J. Weng, Transactions of the ASME 59, 510 (1992)

3. L. Brinson, W. Lin, Composite Structures 41, 353 (1998)

4. F.T. Fisher, L.C. Brinson, Composites Science and Technology 61, 731 (2001)

5. B. Jiang, R. Batra, Journal of intelligent material systems and structures 12, 847 (2001)

6. L. Azrar, A. Bakkali, A.A. Aljinaidi, Composite Structures 113, 281 (2014)

7. A. Bakkali, L. Azrar, A.A. Aljinaidi, Journal of Intelligent Material Systems and Structures (2016)

8. N. Lahellec, P. Suquet, International Journal of Solids and Structures 44, 507 (2007)

9. J.M. Ricaud, R. Masson, International Journal of Solids and Structures 46, 1599 (2009)

10. R. Masson, R. Brenner, O. Castelnau, Comptes Rendus Mécanique 340, 378 (2012)

11. J. Sanahuja, International Journal of Solids and Structures 50, 2846 (2013)

12. M. El Kouri, A. Bakkali, L. Azrar, Applied Mathematical Modelling 40, 4302 (2016)

13. J.F. Barthélémy, A. Giraud, F. Lavergne, J. Sanahuja, International Journal of Solids and Structures 97-98, 530 (2016) 